\title{
Blood pressure and acute kidney injury
}

\author{
Ryota Sato ${ }^{{ }^{*}}$, Sarah Kyuragi Luthe ${ }^{2}$ and Michitaka Nasu ${ }^{1}$
}

\begin{abstract}
Maintaining the optimal blood pressure is an important aspect of preventing acute kidney injury (AKI), especially for vasopressor-dependent patients. Although mean arterial pressure (MAP) has played an important role in previous trials for prevention of AKl, there is little evidence that MAP actually reflects organ perfusion. In fact, several studies have suggested that perfusion pressure, including diastolic perfusion pressure and mean perfusion pressure (MPP) and calculated with central venous pressure (CVP), may be more useful than the widely used MAP to help prevent AKI. This emphasizes the importance of maintaining diastolic arterial pressure and avoiding elevation of CVP to prevent AKI in patients with sepsis or invasive surgery. To achieve this, further investigation regarding titrated fluid therapy and vasopressors is warranted.
\end{abstract}

Keywords: Acute kidney injury, Central venous pressure, Mean arterial pressure, Mean perfusion pressure, Diastolic perfusion pressure

Blood pressure has been considered to be essential for organ perfusion. Therefore, maintaining the optimal blood pressure is an important aspect of preventing acute kidney injury (AKI), especially for vasopressor-dependent patients. Mean arterial pressure (MAP) is widely used as an index for the optimal blood pressure.

However, although the purpose of maintaining MAP is to provide adequate organ perfusion, there is little evidence that MAP actually reflects organ perfusion. Recently, perfusion pressure has been considered as an indicator in the context of preventing AKI. In terms of perfusion pressure, diastolic perfusion pressure (DPP) and mean perfusion pressure (MPP) should be pointed out. These are defined as: $\mathrm{DPP}=$ Diastolic arterial pressure (DAP) - Central venous pressure $(\mathrm{CVP})$; and $\mathrm{MPP}=\mathrm{MAP}$

\footnotetext{
* Correspondence: st051035@gmail.com

${ }^{1}$ Department of Emergency and Critical Care Medicine, Urasoe General

Hospital, 4-16-1, Iso, Urasoe, Okinawa 901-2131, Japan

Full list of author information is available at the end of the article
}

- CVP. A recent observational study revealed that lower DAP and higher CVP were associated with septic AKI, while MAP was not [1]. Another observational study reported that decreased MPP was associated with AKI while MAP was not, suggesting that only CVP may be associated with septic AKI [2]. Furthermore, a recent study of post-cardiac surgery patients showed an association between decreased DAP, MPP, and DPP, but not MAP, and AKI. The decreased DPP was caused by elevation of CVP in $23.8 \%$ of the patients and by decreased DAP in $76.2 \%$ [3].

Although aggressive fluid resuscitation is believed to protect the kidney, this may contribute to the elevation of CVP and consequently AKI as demonstrated above. Previous studies have reported an association between fluid overload and AKI [4]. Additionally, the change in DAP is believed to reflect the alteration of vascular tone. Therefore, since decreased DAP is associated with AKI, vasopressors may be effective for the prevention of AKI [5].

From these perspectives, it is important to maintain DAP and avoid the elevation of CVP to prevent AKI in patients with sepsis or after invasive surgery. To achieve this, further investigation regarding titrated fluid therapy and vasopressors is warranted.

\section{Abbreviations}

AKI: Acute kidney injury; CVP: Central venous pressure; DAP: Diastolic arterial pressure; DPP: Diastolic perfusion pressure; MAP: Mean arterial pressure; MPP: Mean perfusion pressure

\section{Acknowledgements}

Not applicable.

\section{Funding}

None.

Availability of data and materials

Not applicable.

\section{Authors' contributions}

RS was responsible for the conception of the letter and drafted and revised the manuscript. LS and MN helped to draft the manuscript. All authors read and approved the final manuscript. 


\section{Authors' information}

RS is a Japanese board certified emergency physician. LS is a Japanese qualified anesthesiologist. MN is a Japanese board certified emergency and critical care physician.

\section{Competing interests}

The authors declare that they have no competing interest.

\section{Consent for publication}

Not applicable.

\section{Ethics approval and consent to participate}

Not applicable.

\section{Author details}

'Department of Emergency and Critical Care Medicine, Urasoe General Hospital, 4-16-1, Iso, Urasoe, Okinawa 901-2131, Japan. ²Department of Anesthesia, Urasoe General Hospital, Okinawa, Japan.

Received: 9 January 2017 Accepted: 17 January 2017

Published online: 10 February 2017

\section{References}

1. Legrand M, Dupuis C, Simon C, et al. Association between systemic hemodynamics and seotic acute kidney injury in critically ill patients: a retrospective observational study. Crit Care. 2013;17(6):R278.

2. Wong BT, Chan MJ, Glassford NJ, et al. Mean arterial pressure and mean perfusion pressure deficit in septic acute kidney injury. J Crit Care. 2015; 30(5):975-81.

3. Saito S, Uchino S, Takinami M, et al. Postoperative blood pressure deficit and acute kidney injury progression in vasopressor-dependent cardiovascular surgery patients. Crit Care. 2016;20:74.

4. Bouchard J, Soroko SB, Chertow GM, et al. Fluid accumulation, survival and recovery of kidney function in critically ill patients with acute kidney injury. Kidney Int. 2009;76:422-7.

5. Lamia B, Chemla D, Richard C, Teboul JL. Clinical review: interpretation of arterial pressure wave in shock states. Crit Care. 2005;9(6):601-6. 